"This paper is a postprint of a paper submitted to and accepted for publication in IET Radar, Sonar and Navigation and is subject to Institution of Engineering and Technology Copyright. The copy of record is available at IET Digital Library"

\title{
Design of a 24 GHz FMCW Radar System based on Sub-Harmonic Generation
}

\author{
Naglaa El Agroudy ${ }^{1}$, Mohammed El-Shennawy ${ }^{1}$, Niko Joram ${ }^{1}$, Frank Ellinger ${ }^{1}$ \\ ${ }^{1}$ Chair for Circuit Design and Network Theory, Technische Universität Dresden, 01062 Dresden, Germany \\ nnaglaa.elagroudy@tu-dresden.de
}

\begin{abstract}
This paper presents a novel frequency modulated continuous wave (FMCW) radar system based on sub-harmonic generation where a $\mathbf{2 4 ~ G H z}$ frequency divider-by-10 is used as a an active reflector tag. A practical prototype is designed and fabricated on a GF45nm-SOI technology for the $24 \mathrm{GHz}$ building blocks, while a GF0.18 $\mu \mathrm{m}$ 7WL BiCMOS technology was used for the $2.4 \mathrm{GHz}$ receiver (RX) and baseband. System measurement results show that as opposed to conventional primary radars, the proposed system is immune to strong multi-path interferences resulting from direct reflections of the interrogating signal. When measured in lab environment, the system achieves a ranging precision of $3.7 \mathrm{~mm}$. Moreover, when measured in an indoor environment, ranging results show a ranging precision and accuracy of 5.8 and $22.3 \mathrm{~cm}$, respectively, which outperforms other FMCW radars in the literature.
\end{abstract}

\section{Introduction}

Accurate indoor localization systems are highly needed by many applications such as automated vehicles, automotive industries, safe evacuation systems and warehouse management with demanding specifications on accuracy and precision. FMCW radar systems have been proven to be a good candidate for indoor localization systems with a precision of few tens of centimetres [1] and ranging accuracy of few centimetres [2]. However the accuracy of FMCW radar systems is degraded in indoor environments due to multi-path interferences caused by static or moving scatterers.

In order to enhance the robustness of FMCW radar systems in indoor environments against multi-paths, many techniques were adopted. The use of directive antennas was proposed in [1]. Another technique showed that the use of multi-bands for ranging improves the robustness of FMCW radar systems against interferers [3]-[4], however; the added redundancy increases the cost and the processing time. In [5], FMCW radar with spatial diversity was proposed to reduce multi-path interferences resulting from ground reflections in automotive radars. Other approaches to mitigate multi-path interferences are the multiple-input multiple-output (MIMO) radar with digital beam forming (DBF) proposed in [6] and the switched-beam antenna array implemented in [7]. However, the proposed solutions in [5]-[7] come on the expense of more complexity since the base-station (BS) requires either multiple numbers of RXs, mixers or antennas. The use of an artificial on-chip target (OCT) in order to mitigate short range leakage caused from direct reflections of very close objects in front of the radar antennas was investigated in [8]-[10]. This technique is suitable for automotive applications were the car bumpers are usually very close to the radar antennas causing severe interferences. However, in other indoor environments, this technique will not be able to mitigate reflections of moving objects as it depends on designing the delay time of the OCT to be the same as the round-trip delay time of the direct reflections.

The use of modulated backscatter transponders with FMCW radars was also proposed in order reduce multi-path

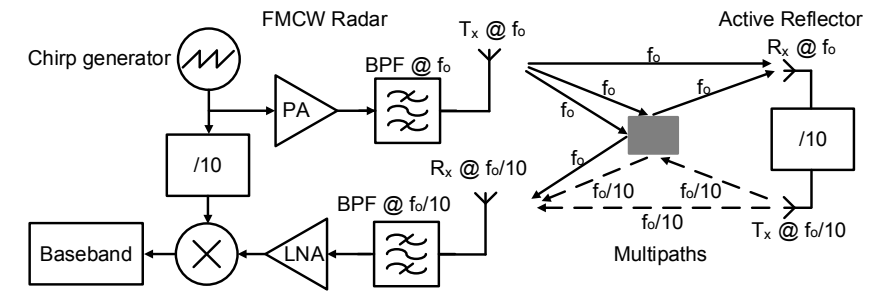

Fig. 1. Proposed FMCW radar system

effects [11]. However, the proposed active tag has a power consumption of $75 \mathrm{~mW}$, which is relatively high for battery operated devices. The use of nonlinear transmission lines (NLTL) as passive harmonic tags was proposed in [12]-[13]. In this system, the transmitter (TX) and the RX of the radar front-end are at two different frequencies. The interrogating signal is transmitted at frequency $f_{0}$ and then reflected at the second $\left(2 f_{0}\right)$ or third $\left(3 f_{0}\right)$ harmonic by the harmonic tag which eliminates multi-path interferences from direct reflections of the interrogating signal. However NLTLs suffer from conversion loss during harmonic generation. Additionally, the low output power of these tags limits the localization range and a high transmitting power of $4 \mathrm{~W}$ is required from the radar BS [13].

In [14], the use of frequency dividers as sub-harmonic generators in FMCW radar systems is proposed in order to eliminate multi-path interferences of the direct reflections of the interrogating signal and to provide a conversion gain of $48 \mathrm{~dB}$ which helps to increase the range of the radar system.

In this work, a first prototype of the FMCW radar system based on sub-harmonic generation is implemented, where the two ISM bands at 24 and $2.4 \mathrm{GHz}$ are utilized for the transmitted and received signals respectively. In this system, the $24 \mathrm{GHz}$ frequency divider-by-10 developed in [14] is used as a sub-harmonic active reflector tag. The rest of this paper is organized as follows; in section 2, an overview of the proposed sub-harmonic FMCW radar system is presented. In section 3 , the design and measurement of both the active reflector tag and the FMCW radar frontend are presented. 
Then in section 4, measurements of the proposed subharmonic FMCW radar system in lab and indoor environments are conducted and compared to state-of-the-art FMCW radars. Finally, conclusions and future work are presented in section 5 .

\section{System Overview}

Fig. 1 shows a top level overview of the proposed subharmonic FMCW radar system. At the TX side, a chirp is generated at a starting frequency $f_{0}$ which is swept linearly over a bandwidth $B_{f m}$ in a time $T_{f m}$, as expressed by:

$$
s_{T X}(t)=A_{T X} \cos \left(2 \pi f_{0}+\frac{B_{f m}}{2 T_{f m}} t^{2}+\varphi_{T X}\right)
$$

where $A_{T X}$ is the signal amplitude at the TX output and $\varphi_{T X}$ is an arbitrary starting phase.

The transmitted chirp $s_{T X}(t)$ is then propagated at the speed of light $c$, till it reaches the sub-harmonic active reflector tag after a time-of-flight (ToF) $\tau$. There, the $10^{\text {th }}$ sub-harmonic is generated by the frequency divider, sent back and received by the RX after the same ToF $\tau$ as expressed by:

$s_{R X}(t)=A_{R X} \cos \left(2 \pi \frac{f_{0}}{10}+\frac{B_{f m} / 10}{2 T_{f m}}(t-2 \tau)^{2}+\varphi_{R X}\right)$

where $A_{R X}$ and $\varphi_{R X}$ are the signal amplitude and phase at the $\mathrm{RX}$ input respectively. The received signal is then mixed with a locally generated $10^{\text {th }}$ sub-harmonic of the transmitted chirp generating a baseband signal whose frequency $f_{D}$ is equal to the frequency difference between the locally generated and received chirps and related to the round-trip time-of-flight (RToF) by:

$$
f_{D}=\frac{0.1 B_{f m} 2 \tau}{T_{f m}}
$$

and then the range $d_{0}$ can be evaluated by:

$$
d_{0}=\frac{f_{D} c T_{f m}}{0.2 B_{f m}}
$$

Since the transmitted and received chirps occupy two different frequency bands, any direct reflections by passive scatterers are not demodulated to a baseband frequency thus leading to better robustness towards multi-path propagation as compared to conventional primary FMCW radars.

\section{System Design}

\subsection{Sub-Harmonic Active Reflector Tags}

A $24 \mathrm{GHz}$ frequency divider-by-10 was designed and fabricated in GF45nm-SOI technology to be used as a subharmonic generator for the active reflector tag [14]. The chip is bonded onto a 4-layer Rogers-4350B printed circuit board (PCB) as shown in Fig. 2. A passive band-pass filter (BPF) and a rat-race balanced-to-unbalanced (balun) converter were designed on the PCB at the $24 \mathrm{GHz}$ input. Voltage regulators and current sources are used to provide the required bias point to the frequency divider. The chip consumes a total current of $7.4 \mathrm{~mA}$ from a $1 \mathrm{~V}$ supply.

The phase noise (PN) of the $2.4 \mathrm{GHz}$ output of the frequency divider is measured at $-128 \mathrm{dBc} / \mathrm{Hz}$ at $1 \mathrm{MHz}$ offset frequency (Fig. 3a). The divider locking range was measured again on PCB after the BPF was used and compared to the previous on-wafer measurements (Fig. 3b).
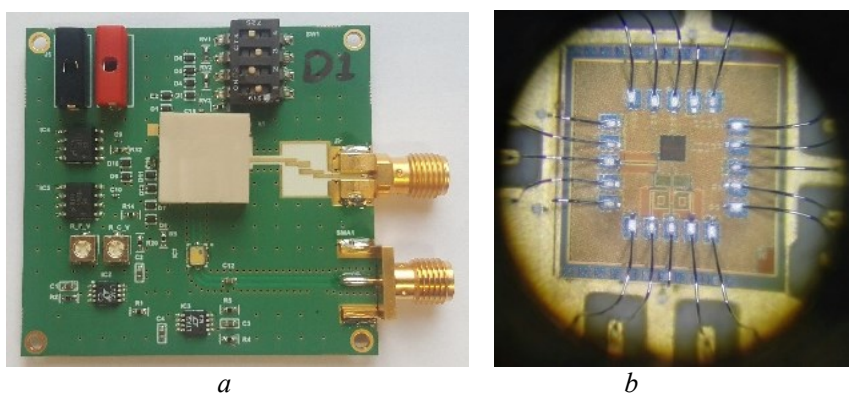

Fig. 2. Active reflector tag

(a) PCB $(4.8 \mathrm{~cm} \times 4.5 \mathrm{~cm})$, (b) Bonded die photo of the frequency divider
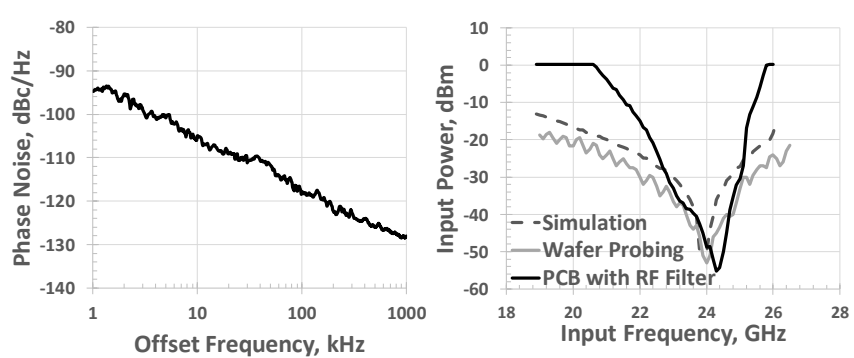

$a$

$b$

Fig. 3. Frequency divider PCB measurements

(a) Phase noise of the frequency divider-by-10 measured on PCB, (b) Divider locking range on PCB with band-pass filter

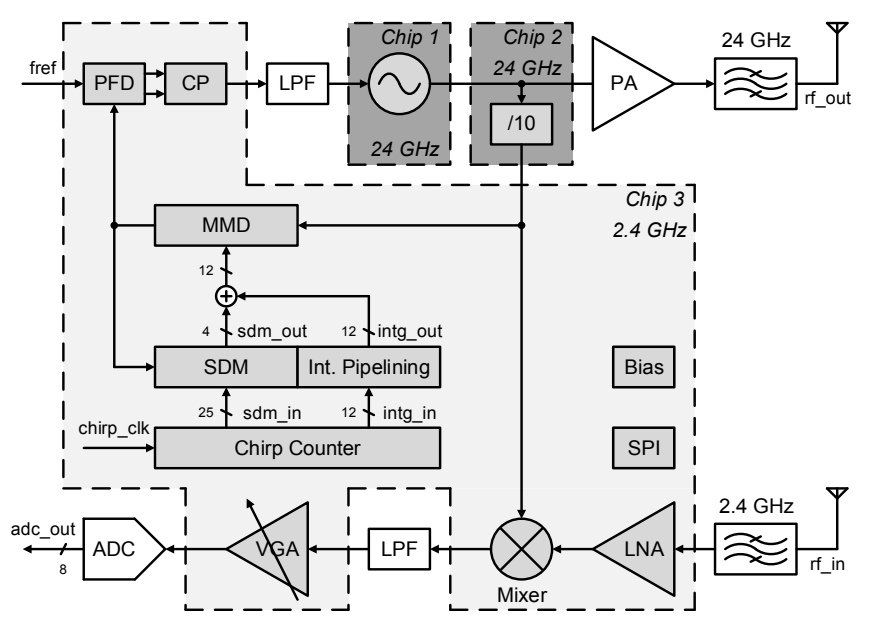

Fig. 4. Sub-harmonic FMCW radar front-end diagram

The PCB measurement still shows a good input sensitivity of $-50 \mathrm{dBm}$ at $24 \mathrm{GHz}$. It is worth noting that since the bond wire inductances were taken into account when designing the on-chip radio frequency (RF) input matching network, the ripples in the on-wafer-probed sensitivity curve of the chip are now smoothed in the PCB measured version.

\subsection{FMCW Radar Front-end}

Fig. 4 shows a simplified block diagram of the proposed sub-harmonic FMCW radar frontend where it is here partitioned into three chips:

Chip 1 is a $24 \mathrm{GHz}$ voltage-controlled oscillator (VCO) that was fabricated also on GF45nm-SOI technology (Fig. 5a). Measured on PCB, the VCO chip has a PN of $-119 \mathrm{dBc} / \mathrm{Hz}$ at $10 \mathrm{MHz}$ offset frequency, an output power of $-10.7 \mathrm{dBm}$ and a $24-26.25 \mathrm{GHz}$ tuning range. 
Chip 2 is the same frequency divider chip used in the active reflector tag. This chip provides the frequency dividedby-10 local oscillator signal for mixing with the $2.4 \mathrm{GHz}$ received signal.

Finally, chip 3 is a $2.4 \mathrm{GHz}$ FMCW radar RX and fractional-N phase locked loop (Frac-N PLL) fabricated on a GF0.18 $\mu \mathrm{m}$ 7WL BiCMOS technology (Fig. 5b). This chip is a modified version of a previous design reported in [15] where on the RX side, it includes the same integrated lownoise amplifier (LNA), mixer, baseband variable gain amplifier (VGA) and automatic gain control (AGC) loop. While on the TX side, the integrated VCO in [15] is replaced by the external $24 \mathrm{GHz} \mathrm{VCO}$ (Chip 1) followed by the $24 \mathrm{GHz}$ frequency divider-by-10 (Chip 2) such that together with the integrated multi-modulus divider (MMD), sigmadelta modulator (SDM), phase frequency detector (PFD) and charge pump (CP), a $24 \mathrm{GHz}$ Frac-N PLL is created. For chirp generation, the fractional (and integer) parts of the PLL frequency control word are incremented gradually using a chirp counter at a rate that is faster than the PLL bandwidth, such that a smooth frequency ramp is generated at the PLL output. In order to mitigate a frequency glitch issue when the output frequency approaches integer multiples of the $20 \mathrm{MHz}$ reference frequency during chirp generation, integer pipelining stages are used as explained in [16].

A summary of the chips specifications is shown in tables 1, 2 and 3. The three chips are bonded on a 4-layer Rogers PCB with dimensions $10 \mathrm{~cm} \times 7 \mathrm{~cm}$ as shown in Fig. 6a. A rat-race balun similar to that used in the active reflector tag is used to convert the differential output of the VCO (Chip 1) to a single-ended output to feed the TX antenna. Additionally, an RF directional coupler is designed on PCB in order to couple the $\mathrm{VCO}$ output to the input of the frequency divider-by-10 (Chip 2). The PCB also includes the PLL loop filter, the baseband low-pass filter (LPF) and the analogue-to-digital converter (ADC) for digitizing the downconverted baseband signal.

Another 8-layer FR-4 PCB (Fig. 6b) with a field programmable gate array (FPGA) and a micro-controller is stacked on top of the radar frontend PCB in order to perform digital signal processing and to provide digital controls.

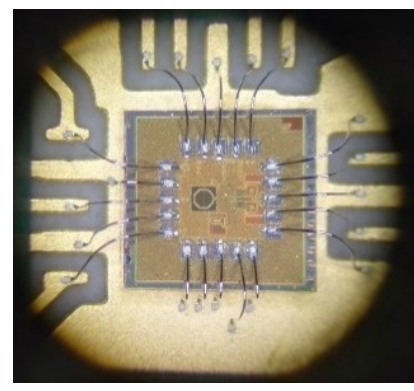

a

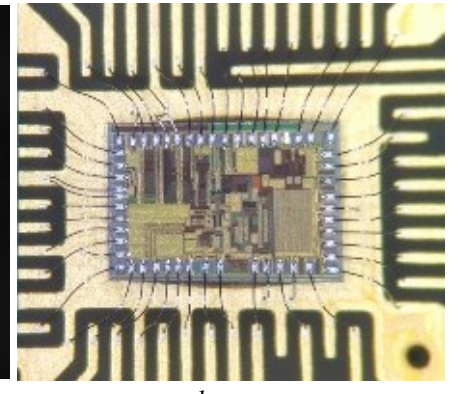

b
Fig. 5. Bonded chip photos

(a) $24 \mathrm{GHz}$ VCO chip, (b) $2.4 \mathrm{GHz}$ FMCW RX and PLL chip
Table 1 Summary of Chip 1 specifications

\begin{tabular}{lcc}
\hline Parameter & Value & Unit \\
\hline Process & $45 \mathrm{SOI}$ & $\mathrm{nm}$ \\
$\mathrm{P}_{\mathrm{dc}}$ & 12.3 & $\mathrm{~mW}$ \\
Supply & 1 & $\mathrm{~V}$ \\
Area & 0.64 & $\mathrm{~mm}^{2}$ \\
Tuning Range & $24-26.5$ & $\mathrm{GHz}$ \\
Efficiency & 2.15 & $\mathrm{GHz} / \mathrm{mW}$ \\
$\mathrm{P}_{\text {out }}$ & -10.7 & $\mathrm{dBm}$ \\
$\mathrm{PN} 10 \mathrm{MHz}$ & -119 & $\mathrm{dBc} / \mathrm{Hz}$ \\
\hline
\end{tabular}

Table 2 Summary of Chip 2 specifications

\begin{tabular}{lcc}
\hline Parameter & Value & Unit \\
\hline Process & $45 \mathrm{SOI}$ & $\mathrm{nm}$ \\
$\mathrm{P}_{\mathrm{dc}}$ & 7.4 & $\mathrm{~mW}$ \\
Supply & 1 & $\mathrm{~V}$ \\
Area & 0.64 & $\mathrm{~mm}^{2}$ \\
Centre Frequency & 24 & $\mathrm{GHz}$ \\
Input Sensitivity Level & -50 & $\mathrm{dBm}$ \\
Relative Bandwidth & 33 & $\%$ \\
Efficiency $^{\mathrm{b}}$ & 3.58 & $\mathrm{GHz} / \mathrm{mW}$ \\
$\mathrm{P}_{\text {out }}$ & -2 & $\mathrm{dBm}$ \\
PN $1 \mathrm{MHz}$ & -128 & $\mathrm{dBc} / \mathrm{Hz}$ \\
\hline
\end{tabular}

a Relative BW $=\frac{\mathrm{f}_{\max }-\mathrm{f}_{\min }}{\left(\mathrm{f}_{\max }+\mathrm{f}_{\min }\right) / 2} \times 100 \%$, at $\mathrm{P}_{\text {in }}=-20 \mathrm{dBm}$

${ }^{\mathrm{b}}$ Efficiency $=\mathrm{f}_{\max } / \mathrm{P}_{\mathrm{dc}}$

Table 3 Summary of Chip 3 specifications

\begin{tabular}{|c|c|c|}
\hline Parameter & Value & Unit \\
\hline Process & $180 \mathrm{BiCMOS}$ & $\mathrm{nm}$ \\
\hline $\mathrm{P}_{\mathrm{dc}}$ & 150 & $\mathrm{~mW}$ \\
\hline Area & 2.4 & $\mathrm{~mm}^{2}$ \\
\hline Frequency Band & 2.4 & $\mathrm{GHz}$ \\
\hline RX Bandwidth & 100 & $\mathrm{MHz}$ \\
\hline RX Conversion Gain & 82.0 & $\mathrm{~dB}$ \\
\hline RX Noise Figure & 7.3 & $\mathrm{~dB}$ \\
\hline RX IP1dB & -24 & $\mathrm{dBm}$ \\
\hline PLL Freq. Resolution ${ }^{\mathrm{a}}$ & 0.6 & $\mathrm{~Hz}$ \\
\hline MMD Divide Ratio (N) & $120-125$ & \\
\hline CP Current & 800 & $\mu \mathrm{A}$ \\
\hline SDM Order & $3^{\text {rd }}$ & \\
\hline SDM Word Width & 25 & bit \\
\hline
\end{tabular}

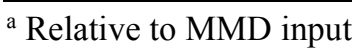

In locked state, the $\mathrm{PN}$ of the generated $24 \mathrm{GHz} \mathrm{VCO}$ signal and the divided-by-10 locally generated signal at $2.4 \mathrm{GHz}$ are measured as shown in Fig. 7, having around $20 \mathrm{~dB}$ difference as expected due to the division factor of 10 . Fig. 8 shows an example of the VCO control voltage $\left(\mathrm{V}_{\text {ctrl }}\right)$ taking the expected triangular shape during the $24 \mathrm{GHz}$ chirp generation. 


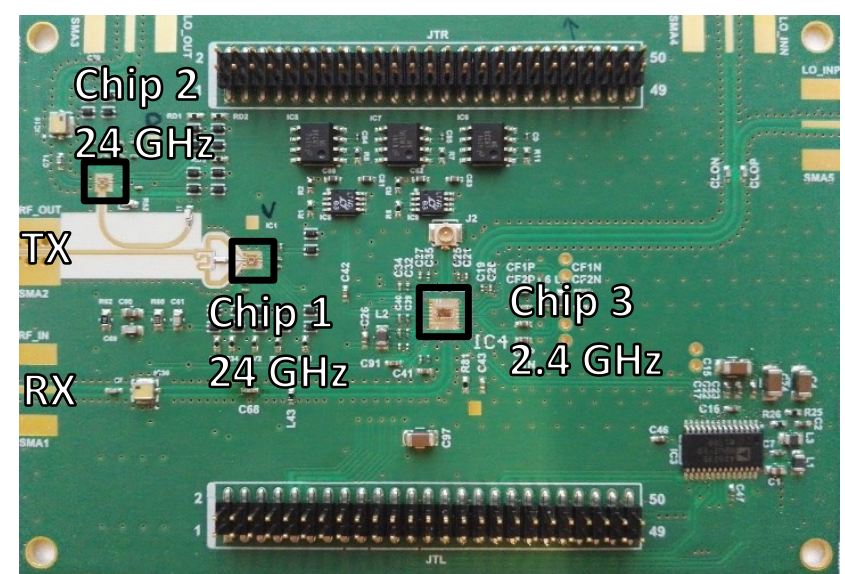

$a$

Fig. 6. FMCW radar base-station

(a) Radar front-end PCB, (b) Digital PCB with FPGA and micro-controller

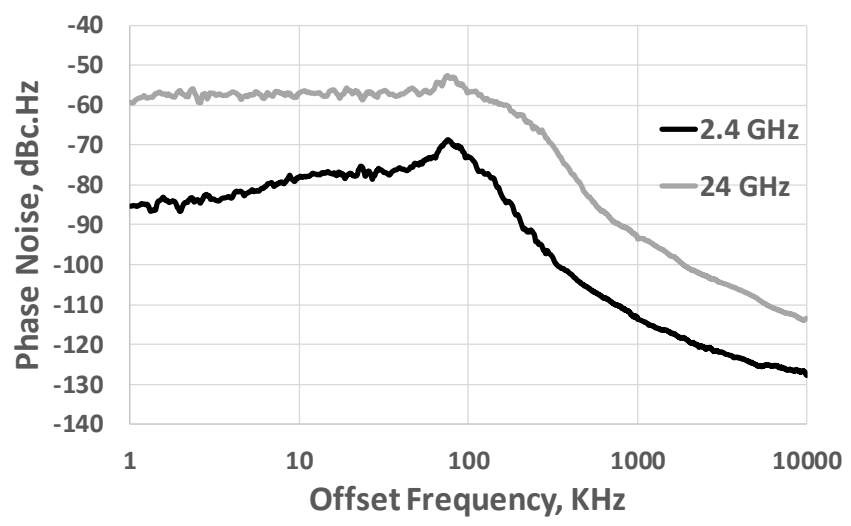

Fig. 7. Measured phase noise of both the fundamental and the local sub-harmonic signal

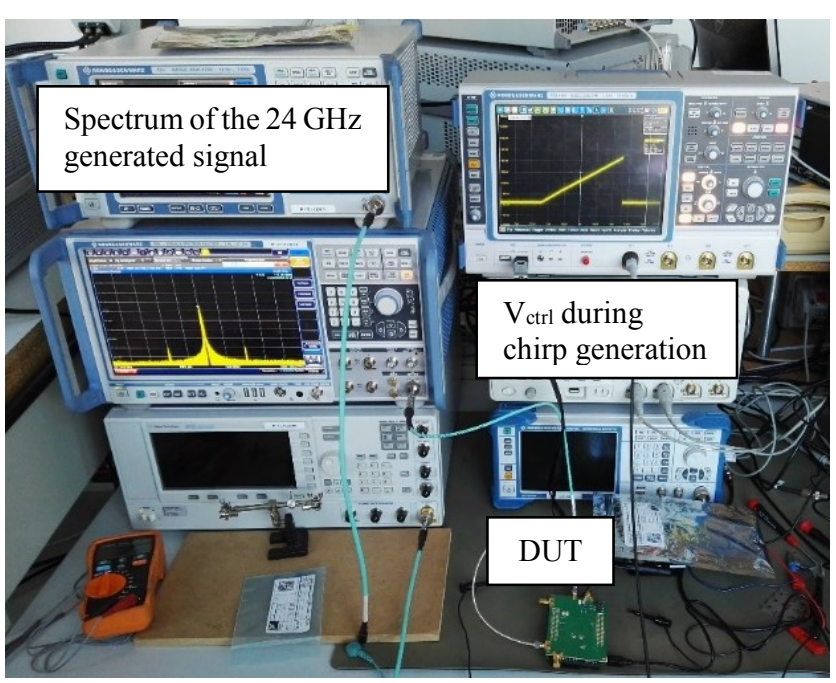

Fig. 8. VCO control voltage during chirp generation

\section{System Measurements}

\subsection{Lab Measurements}

Ranging experiments using the proposed sub-harmonic FMCW radar prototype are performed in laboratory conditions using RF cables and power dividers/combiners in order to easily mimic a direct reflection of the interrogating signal. At the TX side of the FMCW radar frontend, a power divider is used in order to split the power equally into two

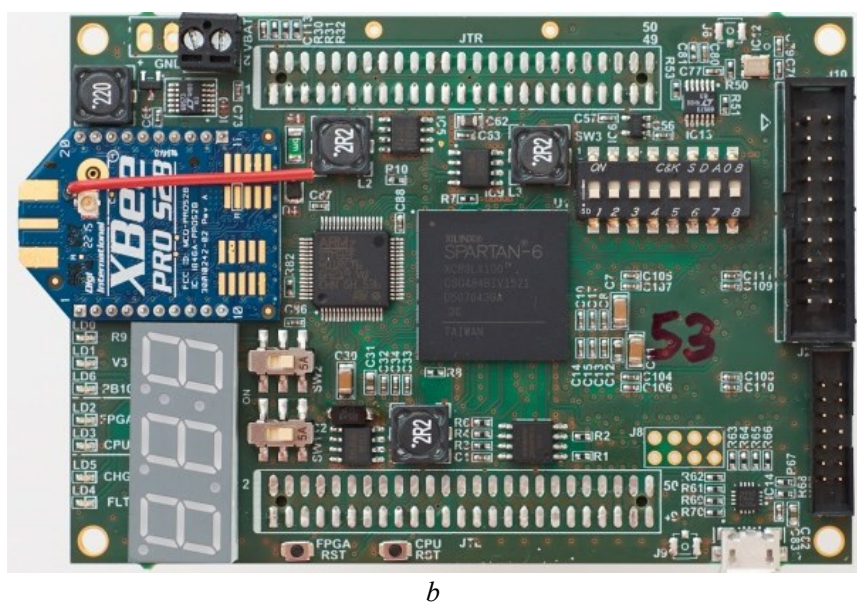

paths, one that is transmitted through a long RF cable to the active tag, and the other is transmitted through a short cable back to the RX side of the FMCW radar front end in order to mimic a strong direct reflection from a passive scatterer as shown in Fig 9a. The directly reflected signal is added through a power combiner to the received subharmonic signal generated from the active tag. For the sake of comparison, a similar setup is used for a conventional primary FMCW radar using the same hardware from [15] at $2.4 \mathrm{GHz}$ as shown in Fig. 9b. Fig. 10 shows the reconstruction of the digitized baseband signal from the sub-harmonic FMCW radar system indicating that chirp generation, transmission, reception and demodulation are successful resulting in a baseband signal with a signal-to-noise ratio of $32 \mathrm{~dB}$ at the $\mathrm{RX}$ output. Fig. 11 shows the final ranging result using both systems. The proposed sub-harmonic FMCW radar system can successfully detect the range to the active tag even with the presence of a strong direct reflection of the interrogating signal (represented by the short RF cable in Fig. 9) while the conventional FMCW radar failed to do so.
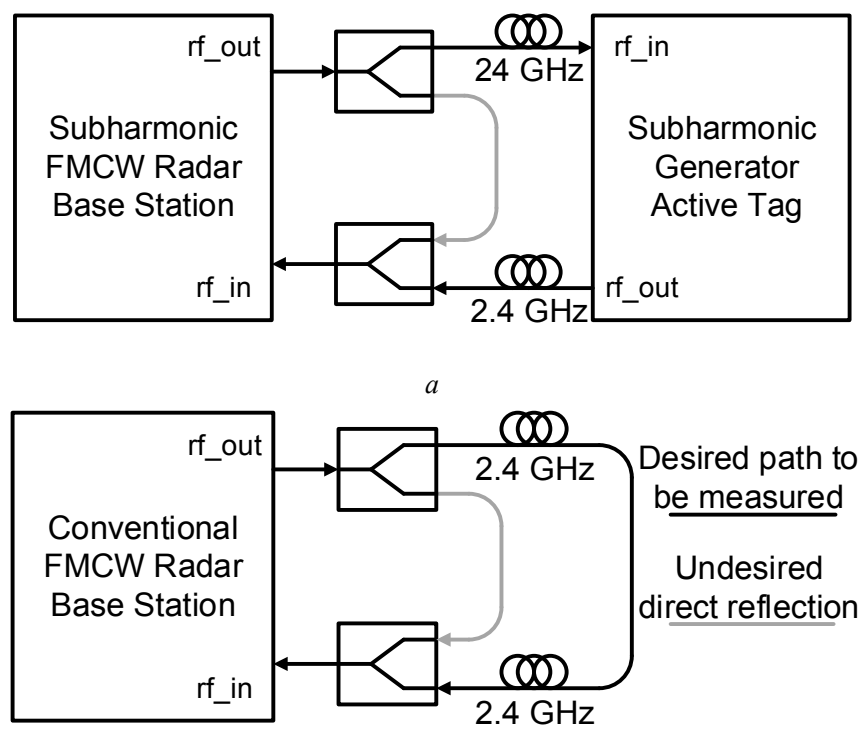

$b$

Fig. 9. Multi-path measurement setup

(a) Sub-harmonic radar ranging, (b) Conventional primary radar ranging 


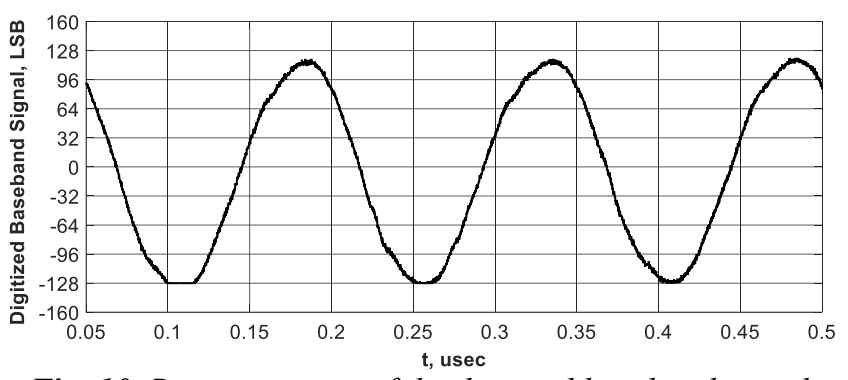

Fig. 10. Reconstruction of the digitized baseband signal

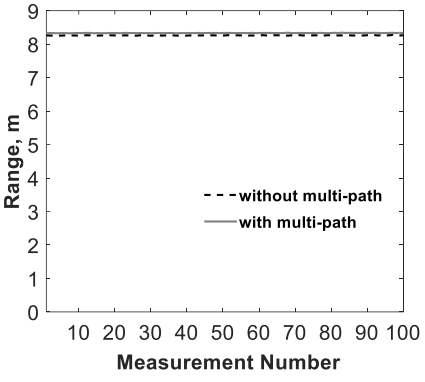

$a$

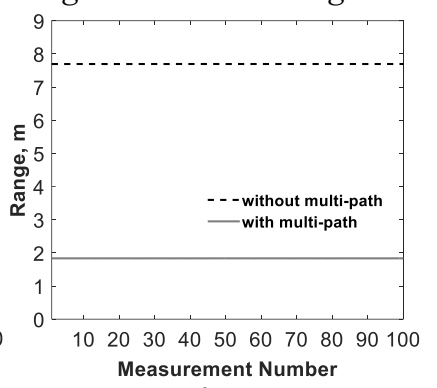

$b$
Fig. 11. Multi-path ranging measurement

(a) Sub-harmonic radar ranging, (b) Primary radar ranging

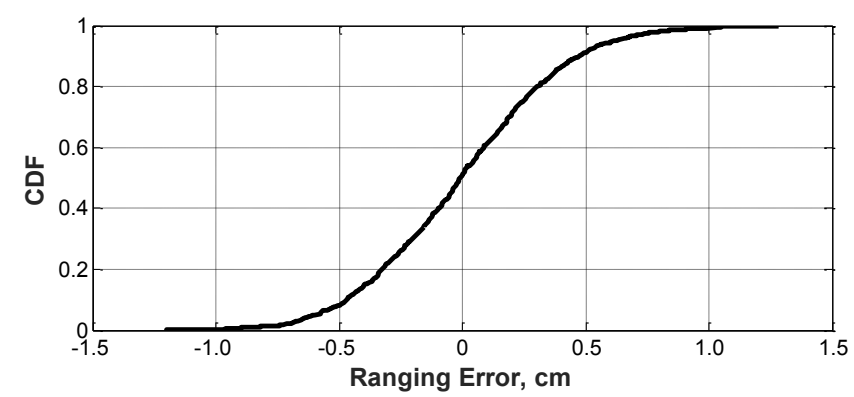

Fig. 12. CDF of the sub-harmonic radar ranging error

A thousand consecutive measurements were performed in order to evaluate the system ranging precision. The corresponding cumulative distribution function (CDF) of the ranging error is plotted in Fig. 12 where the ranging precision is measured at $3.7 \mathrm{~mm}$.

\subsection{Indoor Measurements}

Ranging measurements were conducted indoors as shown in Fig. 13a, where omnidirectional and horn antennas were used for the 2.4 and $24 \mathrm{GHz}$ channels, respectively. The distance between the active tag and the BS was measured at several locations. At each location, one hundred ranging measurements were performed, showing an overall ranging precision of $5.8 \mathrm{~cm}$. Fig. $13 \mathrm{~b}$ shows the measured range calculated by the proposed FMCW radar vs. the actual range acquired from a laser meter. The root-mean-square (RMS) ranging error is measured at $22.3 \mathrm{~cm}$, which shows that the system accuracy is suitable for indoor ranging applications.

In order to further illustrate the system immunity to direct reflections vs. conventional primary radars, another ranging measurement was conducted with a corner reflector as shown in Fig. 14a and b. The proposed system could successfully detect the same desired range as opposed to the primary radar, thus showing its suitability for challenging indoor FMCW radar applications (Fig. 14c and d).
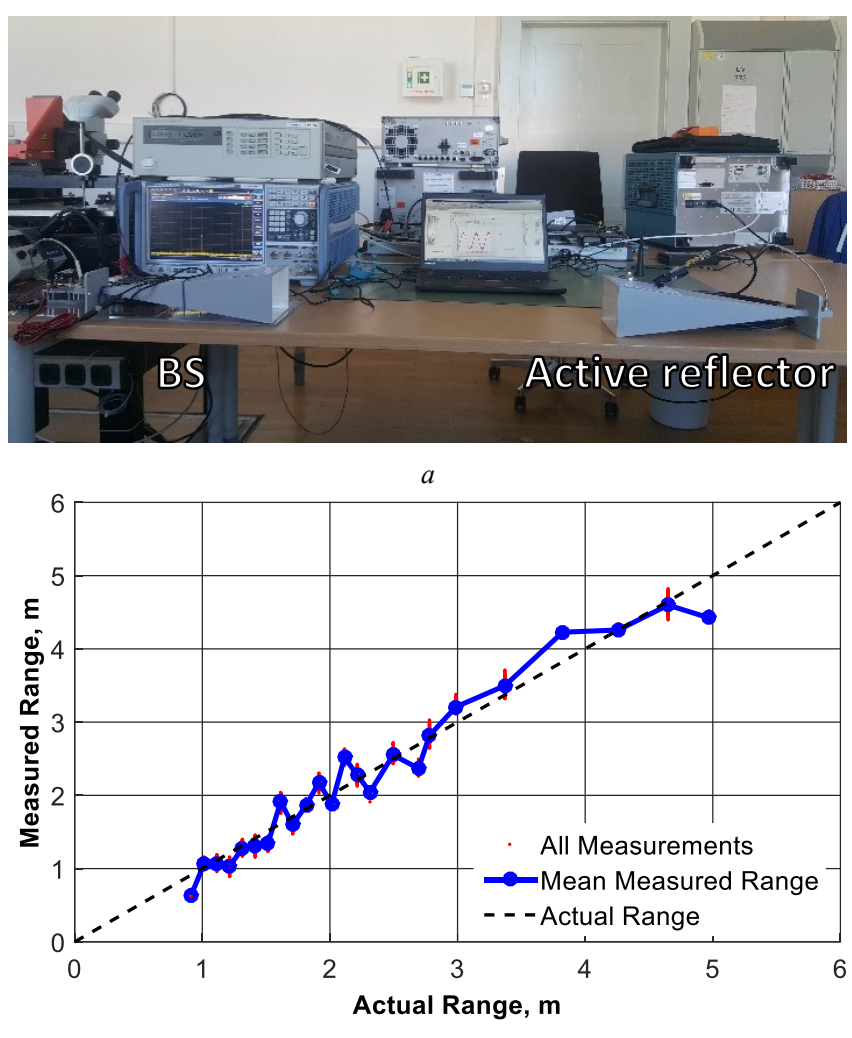

$b$

Fig. 13. FMCW radar indoor measurement results (a) Radar ranging measurement setup (b) Measured range vs. actual range

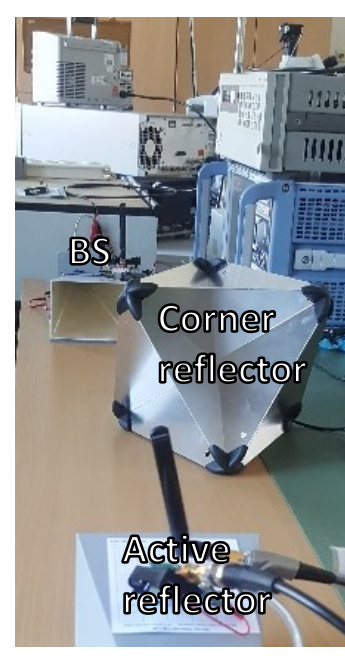

$a$

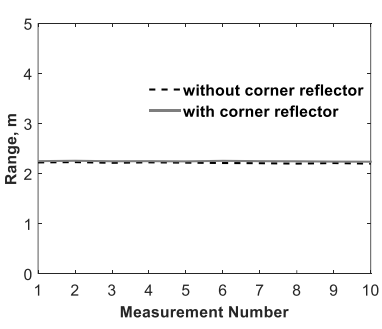

$c$

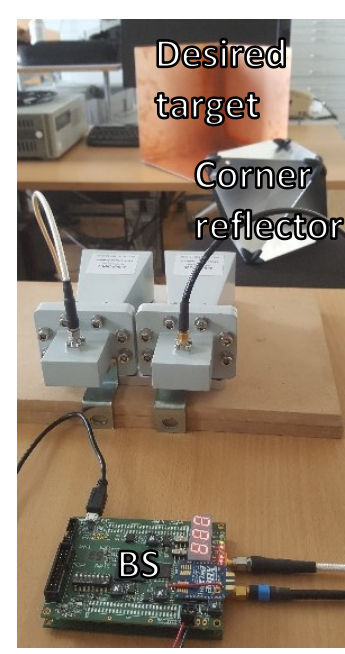

$b$

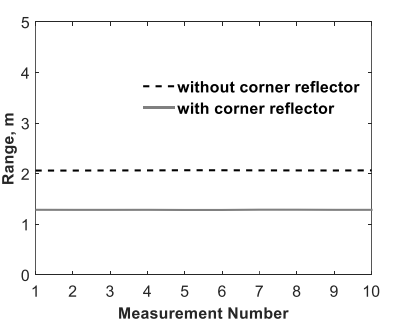

$d$
Fig. 14. FMCW radar measurement results

(a) Subharmonic radar measurement setup with corner reflector (b) Primary radar measurement setup with corner reflector (c) Sub-harmonic radar ranging with and without corner reflector $(d)$ Primary radar ranging with and without corner reflector 
Table 4 Comparison with state-of-the-art FMCW radars

\begin{tabular}{|c|c|c|c|c|c|c|c|}
\hline Parameter & [4] & [6] & [17] & [17] & [18] & This & Unit \\
\hline Frequency & 2.4 & 5.8 & 5.8 & 5.8 & 5.8 & $24 / 2.4$ & $\mathrm{GHz}$ \\
\hline Precision $^{\mathrm{a}}$ & 56 & $\mathrm{~N} / \mathrm{A}$ & 5.96 & 26 & 11.4 & 5.8 & $\mathrm{~cm}$ \\
\hline Accuracy $^{b}$ & 40 & 37.0 & 26.6 & 74.2 & 55 & 22.3 & $\mathrm{~cm}$ \\
\hline Measurement Type & Positioning & Positioning & Ranging & Ranging & Ranging & Ranging & \\
\hline Architecture & Secondary & MIMO & Active Tag & Active Tag & Secondary & Subharmonic Tag & \\
\hline Antenna Type & $\begin{array}{c}\text { Omni- } \\
\text { directional }\end{array}$ & $\begin{array}{l}\text { Four-element } \\
\text { Array }\end{array}$ & Directional & $\begin{array}{c}\text { Omni- } \\
\text { directional }\end{array}$ & Directional & $\begin{array}{l}\text { Directional/Omni- } \\
\text { directional }\end{array}$ & \\
\hline
\end{tabular}

${ }^{a}$ Defined as the standard deviation in a set of ranging measurements taken at the same range

${ }^{\mathrm{b}}$ Defined as the standard deviation of all ranging measurement errors at all ranges

Compared to the state-of-the-art, Table 4 shows that the proposed system outperforms other reported systems in the literature in terms of ranging precision and accuracy. The performance of the proposed system may be further improved by combining the VCO and the frequency divider on one chip leading to a more compact PLL and therefore less susceptibility to noise and interference on the $\mathrm{V}_{\text {ctrl }}$ and supply voltages [19]-[20].

\section{Conclusion and Future Work}

A novel FMCW radar system that is based on subharmonic generation was investigated. Integrated chips required for both RF front-end and the sub-harmonic lowpower active reflector tags were fabricated and the application PCBs were manufactured. System measurements were carried out showing that the proposed system has subcentimetre precision in lab conditions and is immune to interferences caused by direct reflections of the interrogating signal as opposed to conventional primary FMCW radar systems. Future work includes the integration of the VCO and the frequency divider on a single chip, as well as development of more compact $24 \mathrm{GHz}$ patch antennas for the active reflector tags.

\section{Acknowledgment}

The research leading to these results has funding from the European Union's Horizon 2020 under grant agreement $n^{\circ} 636286$ and the European Community's Seventh Framework Program under grant agreement $n^{\circ} 611526$. The authors would like to express their gratitude to GLOBALFOUNDRIES for the fabrication of the $24 \mathrm{GHz}$ circuit prototypes.

\section{References}

[1] Sorrentino, R., Sbarra, E., Urbani, L., Montori, S., Gatti, R. V., Marcaccioli, L.: 'Accurate FMCW radar-based indoor localization system', Proc. IEEE RFID-TA, Nice, Nov. 2012, pp. 362-368.

[2] Mitomo, T., Ono, N., Hoshino, H., Yoshihara, Y.: 'A 77 $\mathrm{GHz} 90 \mathrm{~nm}$ CMOS Transceiver for FMCW Radar Applications', in IEEE JSSC, 2011, 45, (4), pp. 928-937.

[3] Joram, N., Al-Qudsi, B., Wagner, J., Strobel, A., Ellinger, F.: 'Design of a multi-band FMCW radar module', $10^{\text {th }}$ Workshop on Positioning, Navigation and Communication, Dresden, March 2013, pp. 1-6.

[4] Al-Qudsi, B., Joram, N., El-Shennawy, M., Ellinger, F.: 'Scalable indoor positioning system with multi-band FMCW', IET Radar, Sonar \& Navigation, 2018, 12, (1), pp. 46-55.
[5] Wang, H.N., Huang, Y.W., Chung, S.J.: 'Spatial Diversity 24-GHz FMCW Radar with Ground Effect Compensation for Automotive Applications', IEEE Transactions on Vehicular Technology, 2017, 66, (2), pp. 965-973.

[6] Gierlich, R., Huettner, J., Ziroff, A., Weigel, R., Huemer, M.: 'A Reconfigurable MIMO System for High-Precision FMCW Local Positioning', IEEE TMTT, 2011, 59, (12), pp. 3228-3238.

[7] Lee, R.H., Tsai, Z.D., Lang, C.T., Chang, C. C., Chang, S. F.: 'A switched-beam FMCW radar for wireless indoor positioning system', $8^{\text {th }}$ European Radar Conference, Manchester, Oct. 2011, pp. 65-68.

[8] Melzer, A., Huemer, M., Onic, A.: 'Novel mixed-signal based short-range leakage canceler for FMCW radar transceiver MMICs', IEEE ISCAS, Baltimore, MD, May 2017, pp. 1-4.

[9] Melzer, A., Starzer, F., Jäger, H., Huemer, M.: 'RealTime Mitigation of Short-Range Leakage in Automotive FMCW Radar Transceivers', IEEE TCAS II: Express Briefs, 2017, 64, (7), pp. 847-851.

[10] Melzer, A., Onic, A., Starzer, F., Huemer, M.: 'ShortRange Leakage Cancelation in FMCW Radar Transceivers Using an Artificial On-Chip Target', IEEE Journal of Selected Topics in Signal Processing, 2015, 9, (8), pp. 1650-1660.

[11] Lorenzo, J., Lazaro, A., Villarino, R., Girbau, D.: 'Active Backscatter Transponder for FMCW Radar Applications', IEEE Antennas and Wireless Propagation Letters, 2015, 14, pp. 1610-1613.

[12] Yu, F., Lyon, K., Kan, E.: 'A novel passive RFID transponder using harmonic generation of nonlinear transmission lines', IEEE TMTT, 2010, 58, (12), pp. 4121-4127.

[13] Ma, Y., Kan, E.: 'Accurate indoor ranging by broadband harmonic generation in passive NLTL backscatter tags', IEEE TMTT, 2014, 62, (5), pp. 1249 1261.

[14] El Agroudy, N., El-Shennawy, M., Joram, N., Ellinger, F.: 'Design of a $24 \mathrm{GHz}$ frequency divider-by-10 in 45 $\mathrm{nm}$-silicon-on-insulator as an active reflector tag', IET Electronics Letters, 2017, 53, (23), pp. 1546-1548.

[15] El-Shennawy, M., Al-Qudsi, B., Joram, N., Ellinger, F.: 'A dual band FMCW radar receiver with integrated active balun and baseband AGC loop', IEEE ISCAS, Baltimore, MD, May 2017, pp. 1-4.

[16] El-Shennawy, M., Joram, N., Ellinger, F.: 'Fractional-N PLL optimization for highly linear wideband chirp generation for FMCW radars', Proc. GeMiC, Nuremberg, March 2015, pp. 248-251. 
[17] Wehrli, S., Gierlich, R., Huttner, J., Barras, D., Ellinger, F.: 'Integrated Active Pulsed Reflector for an Indoor Local Positioning System', IEEE TMTT, 2010, 58, (2), pp. 267-276.

[18] Joram, N., Wagner, J., Strobel, A, Ellinger, F.: '5.8 GHz demonstration system for evaluation of FMCW ranging', $9^{\text {th }}$ Workshop on Positioning, Navigation and Communication, Dresden, March 2012, pp. 137-141.

[19] Shimizu, S., Mizuno, J., Morishita, S., Hida, K., Yoshimura, T.: 'Analysis and modeling of oscillators with interference noise', IEEE ICECS, Marseille, Feb. 2014, pp. 128-131.

[20] Namgoong, W.: 'An All-Digital Approach to Supply Noise Cancellation in Digital Phase-Locked Loop', IEEE Transactions on Very Large Scale Integration Systems, 2016, 24, (3), pp. 1025-1035. 